Check for updates

Cite this: RSC Adv., 2017, 7, 18765

Received 12th December 2016

Accepted 21st March 2017

DOI: $10.1039 / c 6 r a 28063 c$

rsc.li/rsc-advances

\section{Preparation and characterization of surface molecularly imprinted films coated on multiwall carbon nanotubes for recognition and separation of lysozyme with high binding capacity and selectivity $\dagger$}

\author{
Xinya Xu, Pengqi Guo, Zhimin Luo, Yanhui Ge, Yulan Zhou, Ruimiao Chang, Wei Du, \\ Chun Chang and Qiang Fu (D) *
}

In this work, a series of facile and efficient molecularly imprinted polymers (MIPs) for the selective recognition and separation of lysozyme were synthesized by combining self-polymerization and nanosized matrix. The imprinted materials containing recognition sites for the lysozyme were formed via using both carboxyl-functionalized multi-walled carbon nanotubes (MWCNTs-COOH) as a support and dopamine (DA) with excellent biocompatibility as a functional monomer. The obtained polymers were characterized and evaluated by using field-emission scanning electron microscopy (FESEM), fieldemission transmission electron microscopy (FETEM), nitrogen physisorption experiments, Fourier transform infrared (FT-IR) spectroscopy and thermogravimetric analysis (TGA). The optimum reaction conditions and adsorption performance of the resultant nanomaterials were also investigated. MIPs synthesized by this method exhibited excellent imprinting factor (4.1) and high binding capacity (418 mg $\mathrm{g}^{-1}$ ) for lysozyme. After six adsorption-desorption cycles, the adsorption capacity of the MIPs was only reduced by $7.4 \%$. In addition, the prepared MIPs were used to separate and condense lysozyme from chicken egg white successfully, which showed potential values in industrial protein purification, basic biomedical research and clinical diagnostics.

\section{Introduction}

Lysozyme ( $N$-acetylmuramide glycanohydrolase) is a natural self-defense enzyme present in a wide variety of locations, including egg white, tears, serum and many organs of vertebrates. It can specifically hydrolyze the mucopolysaccharides in bacterial cell walls without any toxicity to human beings. Lysozyme has been used as a harmless antibacterial agent in food chemistry and a drug for treatment of infections, ulcers and cancers, since it has antiviral and anti-inflammatory activities. ${ }^{\mathbf{1}, 2}$ Commercially purified lysozyme could be obtained through various complex and time consuming processes, such as ultrafiltration, precipitation, ion exchange and chromatographic separation..$^{3-5}$ However, the wide applications of these methods are limited by time-consuming processes, low binding efficiency and expensive equipment. Therefore, the design of more efficient, specific and economical strategies has attracted

School of Pharmacy, Xi'an Jiaotong University, Xi'an 710061, PR China. E-mail: fuqiang@mail.xjtu.edu.cn; Tel: +86 2982655382

$\dagger$ Electronic supplementary information (ESI) available. See DOI: $10.1039 / \mathrm{c} 6 \mathrm{ra} 28063 \mathrm{c}$ increasing research interest in lysozyme separation and purification from a complex matrix.

Molecular imprinting is considered as a relatively modern technique for the separation and analysis of specific compound in complex matrix. For this purpose, specific binding sites, known as synthetic receptors, are created on the surface of polymer network. It involves the polymerization of functional monomer and cross-linker in the presence of desired template and the subsequent removal of the template from the resulting polymers. ${ }^{6,7}$ Molecularly imprinted polymers (MIPs) have attracted great interest in terms of its applications in separation processes (chromatography, solid phase extraction, membrane separations), medical diagnostics, environmental monitoring and drug release/delivery. ${ }^{8-10}$ Advantages of using MIPs include easy preparation, low cost, predictable specific recognition, high specificity and high mechanical/chemical stability. ${ }^{11,12}$ Although the imprinting of small molecules has been well established and some have been developed to an industrial scale ${ }^{13}$ high-molecular-weight protein imprinting still faces many challenges. The major difficulties include (a) the insolubility of proteins in commonly utilized imprinting solvents; (b) restricted mobility due to large molecular size and structural complexity; (c) the degradation of proteins under 
polymerization conditions such as high temperature. ${ }^{\mathbf{1 4 - 1 6}}$ Diverse approaches have been proposed to solve these difficulties, such as moderately cross-linked hydrogels, metal coordination, microcontact imprinting, epitope imprinting and surface imprinting. ${ }^{15-23}$ Among the above methods, surface imprinting with the recognition cavities located on the surface of support materials has received more attention.

Surface imprinting method, which distributes the binding sites on the substrate surface, is considered to be an efficient way to overcome the restricted mass transfer and increase the binding affinity of the template. ${ }^{24}$ Among the various nanosized substrates, multiwalled carbon nanotubes (MWCNTs), ever since their discovery, have formed a part of extensive and multidisciplinary research due to their superior properties and wide range of applications over other materials. The unique properties of MWCNTs include, a high tensile strength (which can be 100 times more than that of stainless steel), good biocompatibility, good chemical stability and practical ability to establish different types of interactions with both organic and inorganic analytes. Moreover, MWCNTs have nm-sized diameters and $\mu \mathrm{m}$-sized lengths, so they have around 1000 of the length-to-diameter ratio, resulting in large surface area and high absorption capacity. These properties determine that carbon nanotubes could be used as a good support material in separation science. When MWCNTs are used as MIPs supporting material, the binding sites of imprinting materials located in the outer layer of the composite. This fact is clearly opposed to that of traditional MIPs where the generated cavities could not be in the surface or partly embedded in the imprinting layer. ${ }^{25-27}$ Thus, the imprinting nano-structured materials can provide better site accessibility, well-defined shape, lower mass transfer resistance, and more complete removal of templates than traditional methods so that the high binding capacity and fast binding kinetics can be expected. ${ }^{28,29}$ In order to improve the weak interfacial adhesion, several functional groups (i.e., amino or vinyl group) were introduced to the surface of carbon nanotubes by covalent bonding to provide strong interfacial interactions between MWCNTs and MIPs. ${ }^{30-32}$ These polymerizations often involve multiple reaction steps under harsh conditions, which are inappropriate for the imprinting of biological molecules. Therefore, a simple and efficient chitosan (CS) modification strategy was used to improve the dispersion stability of MWCNTs and to increase the number of adsorption sites on MWCNTs for target protein. Moreover, a green and simple functional monomer was proposed to synthesize molecularly imprinted polymers. It is well known that dopamine (DA) with multifunctional groups shows excellent filmforming ability onto different kinds of materials in weak base solution at room temperature. ${ }^{33,34}$ Considering the solubility and complicacy of protein, dopamine (DA) was used as functional monomer and crossing linker to form imprinted shell on the surface of MWCNTs in this work.

Combining the advantages of MWCNTs and DA, we demonstrate a rather facile and effective strategy to imprint protein through self-polymerization and nanotechnology. The imprinted nanomaterial was obtained just by mixing MWCNTs, dopamine and template protein in weak alkaline aqueous solution.
The preparation procedures were much simpler, and the whole process was highly suitable for the concept of "Green Chemistry”. Field emission scanning electron microscopy (FESEM), field-emission transmission electron microscopy (FETEM), Fourier transform infrared (FT-IR) spectroscopy and thermogravimetric analysis (TGA) were used to characterize the desired products. The adsorption properties of the prepared polymers were investigated by isothermal rebinding, dynamic adsorption and selectivity experiment. In addition, the practicability for biological application was further assessed by separation and enrichment of lysozyme from chicken egg white. Considering to the advantages of the proposed imprinting polymers, such as high selectivity, excellent biocompatibility, good practicability and especially simple operation processes, it could potentially broaden the applicability of imprinting technique to the enrichment and purification of protein in real samples.

\section{Experimental}

\subsection{Materials and reagents}

Carboxyl-functionalized multi-walled carbon nanotubes (outer diameter 20-30 nm, -COOH content $2.00 \mathrm{wt} \%$ ) was purchased from Beijing DK Nano Technology Co., Ltd. (China). Dopamine hydrochloride was obtained from J\&K Scientific Ltd. (China). Chitosan (CS) with $90 \%$ degree of deacetylation $\left(M_{\mathrm{W}}=\right.$ $600000 \mathrm{~g} \mathrm{~mol}^{-1}$ ) were purchased from Sinopharm Chemical Reagent Co., Ltd. Bovine serum albumin (BSA), human serum albumin (HSA) and pepsin were obtained from Sigma-Aldrich. Lysozyme (Lyz) and bromelain (BR) were purchased from J\&K Scientific Ltd. (China). Absolute acetic acid (99\%), $N, N, N^{\prime}, N^{\prime}$ tetramethylethylenediamine (TEMED), sodium dodecyl sulfate (SDS) and other chemicals were purchased from Sinopharm Chemical Reagent Co., Ltd. All other chemicals were of analytical reagent grade and provided by local suppliers.

\subsection{Preparation of Lyz-MIPs and NIPs}

The general scheme for the synthesis of imprinting polymers for lysozyme (Lyz-MIPs) is shown in Fig. 1. The chitosan-wrapped carboxyl-functionalized multi-walled carbon nanotubes (MWCNT-COOH@CS) were prepared as follows: MWCNTs$\mathrm{COOH}(300 \mathrm{mg}$ ) was dispersed in $40 \mathrm{~mL}$ of water and sonicated for $12 \mathrm{~h}$ to obtain a uniform dispersion of MWCNTs-COOH solution. Then, $30 \mathrm{~mL}$ of $1 \%$ HAc solution containing $0.04 \mathrm{~g}$ of chitosan (CS) was slowly added dropwisely into the suspension with constant mechanical stirring at room temperature. The reaction mixture was stirred for further $4 \mathrm{~h}$ at $40{ }^{\circ} \mathrm{C}$, after that, the suspension was separated by centrifugation and washed 3 times using deionized water. The resulting powder was dried under vacuum at $60{ }^{\circ} \mathrm{C}$ for $12 \mathrm{~h}$. The Lyz-MIPs was prepared as follows: MWCNT-COOH@CS (0.1 g) was dispersed in $10 \mathrm{~mL}$ of Tris-HCl buffer (pH 8.5, $50 \mathrm{mM}$ ) by stirring for $30 \mathrm{~min}$. Then Lyz (35 mg) was added with consecutive stirring for $3 \mathrm{~h}$ to obtain the pre-assembled solution. After adding $0.06 \mathrm{~g}$ of DA, the mixed solution was stirred for another $12 \mathrm{~h}$ at $30{ }^{\circ} \mathrm{C}$. The resulting products were washed with SDS-HAc (2\%) to remove the embedded template protein until no adsorption was detected by 

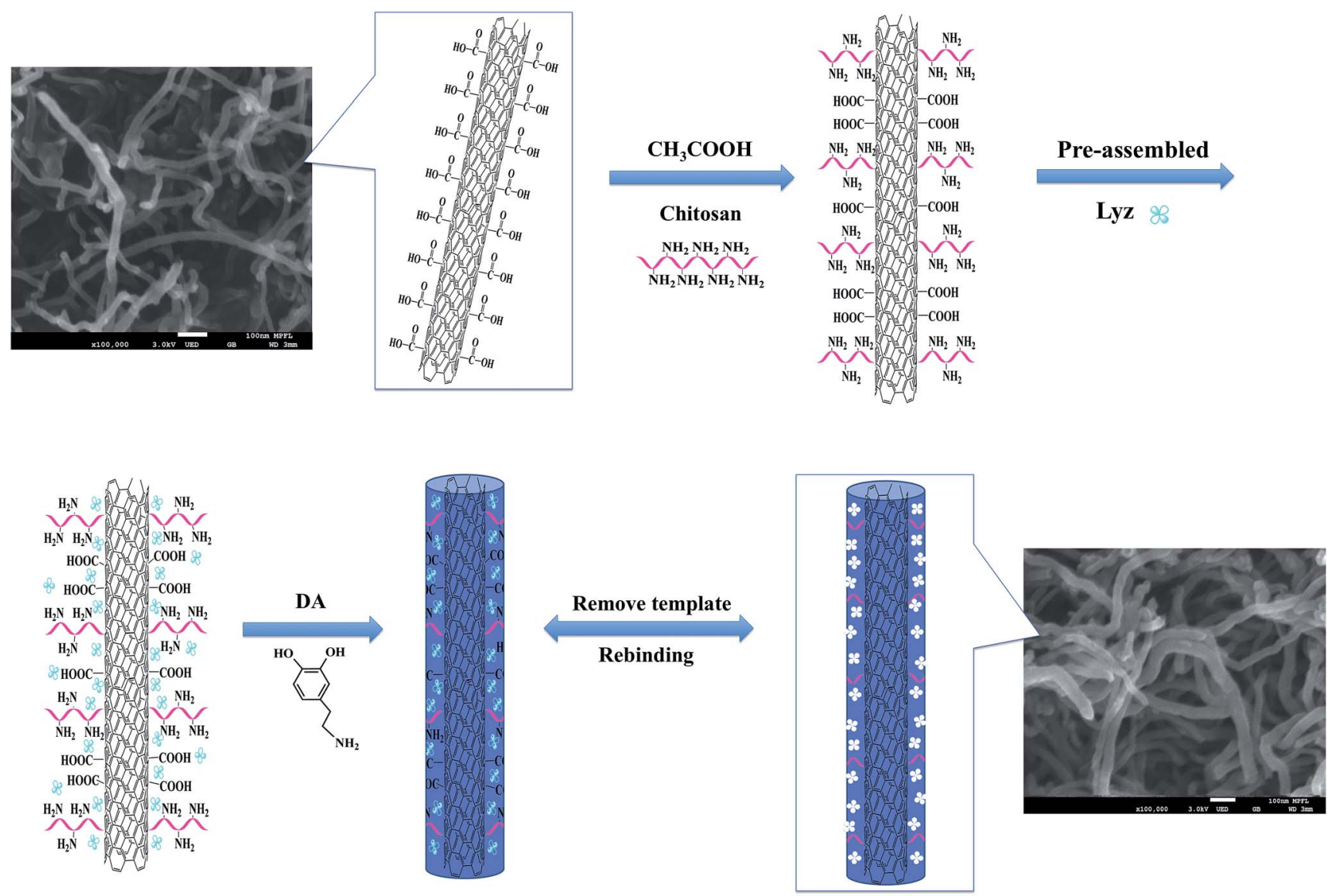

Fig. 1 Schematic of the synthetic route used to prepare Lyz-MIPs.

UV-Vis spectrophotometer at $280 \mathrm{~nm}$. The obtained Lyz-MIPs were centrifuged and washed with ultra pure water until the $\mathrm{pH}$ was shown to be neutral, and then dried under vacuum at $60{ }^{\circ} \mathrm{C}$ for $24 \mathrm{~h}$. For comparison, non-imprinted nanomaterials (denoted as NIPs) were prepared following the same procedure in the absence of the template protein Lyz.

\subsection{Characterization}

The morphologies of the Lyz-MIPs and NIPs were observed by a G2F30 $300 \mathrm{kV}$ field-emission transmission electron microscopy (FETEM) (FEI, USA) and a JEOL 7800F field emission scanning electron microscopy (FESEM) (Hitachi, Japan). The surface areas of the polymers was measured by nitrogen adsorption and desorption experiments using an Autochem 2920 physical chemistry analyzer (Quantachrome, USA). The chemical compositions of the obtained materials were recorded by an FTIR-8400S spectrometer (Shimadzu, Japan) with a scanning range from 400 to $4000 \mathrm{~cm}^{-1}$ at room temperature. Thermo gravimetric analysis (TGA) of the Lyz-MIPs and NIPs were performed with an SDTQ600 thermogravimetric analyzer (TA, New Castle, USA) with ramp $10{ }^{\circ} \mathrm{C} \min ^{-1}$ from room temperature to $800{ }^{\circ} \mathrm{C}$.

\subsection{Adsorption experiments}

All the binding experiments were carried out in a triangular flask by using a batch technique. Before binding experiments, a calibration curve was obtained from the UV-Vis spectra of the Lyz solutions with different concentrations.

The adsorption kinetic experiments were performed as follows: $10 \mathrm{mg}$ of Lyz-MIPs or NIPs was dispersed in $10 \mathrm{~mL}$ of Tris-HCl buffer solution ( $\mathrm{pH} 8.5$ ) with $0.60 \mathrm{mg} \mathrm{mL}^{-1}$ of Lyz. The flasks were shaken at $30{ }^{\circ} \mathrm{C}, 190 \mathrm{rpm}$ for $90 \mathrm{~min}$, then the mixture was centrifuged and the supernatant solution was collected. The maximum absorbances of Lyz in the supernatant before and after adsorption were measured by UV-Vis spectrophotometer at $280 \mathrm{~nm}$ with Tris-HCl buffer solution $(\mathrm{pH} \mathrm{8.5,} 50$ $\mathrm{mM})$ as the blank. The adsorption amount of $\operatorname{Lyz}\left(Q_{t}, \mathrm{mg} \mathrm{g}^{-1}\right)$ at time $t$ ( $\mathrm{min}$ ) was calculated according to the following equation:

$$
Q_{t}=\frac{\left(C_{0}-C_{t}\right) V}{m}
$$

where $C_{0}\left(\mathrm{mg} \mathrm{mL}^{-1}\right)$ is the initial Lyz concentrations; $C_{t}(\mathrm{mg}$ $\left.\mathrm{mL}^{-1}\right)$ is the free Lyz concentration at time $t ; V(\mathrm{~mL})$ and $m(\mathrm{~g})$ are the volume of solution and mass of nano-adsorbent, respectively.

The isothermal adsorption experiments were proceeded as follows: $10 \mathrm{mg}$ of Lyz-MIPs or NIPs was added to $10 \mathrm{~mL}$ of Tris$\mathrm{HCl}$ buffer solution ( $\mathrm{pH} 8.5,50 \mathrm{mM})$ with varied initial concentration (0.10-0.70 $\left.\mathrm{mg} \mathrm{mL} \mathrm{mL}^{-1}\right)$ of Lyz. The adsorption experiments were carried out at $30{ }^{\circ} \mathrm{C}$ for $1 \mathrm{~h}$ to reach equilibrium. The residual Lyz concentration in the supernatant was measured by UV-Vis spectrophotometer at $280 \mathrm{~nm}$. The 
equilibrium adsorption capacity $Q_{\mathrm{e}}\left(\mathrm{mg} \mathrm{g}^{-1}\right)$ of Lyz was calculated according to the following equation:

$$
Q_{\mathrm{e}}=\frac{\left(C_{0}-C_{\mathrm{e}}\right) V}{m}
$$

where $C_{0}\left(\mathrm{mg} \mathrm{mL}^{-1}\right)$ and $C_{\mathrm{e}}\left(\mathrm{mg} \mathrm{mL}^{-1}\right)$ are the initial and equilibrium Lyz concentrations, respectively; $V(\mathrm{~mL})$ and $m(\mathrm{~g})$ are the volume of solution and mass of nanoadsorbent, respectively.

To evaluate the selective adsorption ability, $10 \mathrm{mg}$ of LyzMIPs or NIPs was added to $10 \mathrm{~mL}$ of Tris-HCl buffer solution (pH 8.5, $50 \mathrm{mM}$ ) containing $0.3 \mathrm{mg} \mathrm{mL}^{-1}$ of BSA, HSA, BR and pepsin, respectively. The mixture was shaken at $30{ }^{\circ} \mathrm{C}, 190 \mathrm{rpm}$ for $90 \mathrm{~min}$. After that the extraction and detection procedures were conducted according to the method, which was described in the kinetic adsorption experiments. The imprinting factor $(I F)$ and selectivity coefficient $(S C)$ were used to evaluate the selectivity properties of Lyz-MIPs or NIPs toward the template protein and competitive protein. The $I F$ and $S C$ were calculated from the following equations:

$$
\begin{gathered}
I F=\frac{Q_{\mathrm{MIP}}}{Q_{\mathrm{NIP}}} \\
S C=\frac{I F_{\mathrm{t}}}{I F_{\mathrm{c}}}
\end{gathered}
$$

where $Q_{\text {MIP }}$ and $Q_{\text {NIP }}\left(\mathrm{mg} \mathrm{g}^{-1}\right)$ represent the adsorption capacity of Lyz on Lyz-MIPs and NIPs, respectively; $I F_{\mathrm{t}}$ and $I F_{\mathrm{c}}$ are the imprinting factors for template protein and competitive protein, respectively.

\subsection{Regeneration of Lyz-MIPs and NIPs}

In order to test the reusability of Lyz-MIPs and NIPs, adsorption-desorption procedure for Lyz was repeated for 6 times by using the same polymers. For this purpose, $10 \mathrm{mg}$ of Lyz-MIPs and NIPs were added to $10 \mathrm{~mL}$ of Lyz solution at a concentration of $0.30 \mathrm{mg} \mathrm{mL}^{-1}$ and incubated at room temperature for $1 \mathrm{~h}$. Then, the polymers were separated by centrifugation and the concentration of Lyz in supernatant was quantified by UVVis spectrophotometer. The reused polymers were washed by SDS-HAc (2\%) and purified water to remove the residual Lyz in the polymers. The recovered imprinting nanomaterials were used for another succeeding five adsorption-regeneration cycles to evaluate the reusability of Lyz-MIPs.

\subsection{Application in real samples}

The prepared Lyz-MIPs and NIPs were applied to purify Lyz from chicken egg white. The egg white was collected from fresh eggs and diluted to $50 \%(\mathrm{v} / \mathrm{v})$ with Tris- $\mathrm{HCl}$ buffer solution $(\mathrm{pH} 8.5$, $50 \mathrm{mM}$ ). The mixture was mechanically agitated in an ice bath for $30 \mathrm{~min}$, and then centrifuged at $4{ }^{\circ} \mathrm{C}$ and $10000 \mathrm{rpm}$ for $30 \mathrm{~min}$. The supernatant fluid was diluted 10 -fold and used as the source of lysozyme.

Twenty milligrams of Lyz-MIPs or NIPs were added to $10 \mathrm{~mL}$ of the prepared chicken white solution. After incubation at $25{ }^{\circ} \mathrm{C}$ for $30 \mathrm{~min}$, the suspension was centrifuged at $4000 \mathrm{rpm}$ for $15 \mathrm{~min}$. The polymers were gently treated with Tris-HCl buffer solution ( $\mathrm{pH} 8.5,50 \mathrm{mM}$ ) to remove the weakly adsorbed proteins. Then, the strongly adsorbed proteins were exhaustedly washed by $0.1 \mathrm{M} \mathrm{NaCl}(2 \%)$ solution. The diluted, adsorbed and eluted samples were characterized by using SDS-PAGE.

\section{Results and discussion}

\subsection{Preparation and characterization of Lyz-MIPs}

Fig. 1 shows a schematic diagram of the synthetic route that was used to prepare the imprinting polymers (denoted as Lyz-MIPs). Firstly, chitosan was introduced to the surface of MWCNTs$\mathrm{COOH}$ through oscillation and ultrasonication steps. The trace-chitosan-wrapped multi-walled carbon nanotubes structure can reduce the strong interactions among these nanotubes, prevent their aggregation and increase the number of adsorption sites for target protein. Then, a thin adherent PDA layer embedding Lyz on the surface of MWCNTs-COOH@CS was formed by a one-step spontaneous deposition of dopamine in weak alkaline solution. Because dopamine contains both amino and hydroxyl groups, these functional groups could interact with Lyz through multi-hydrogen bond and hydrophobic interaction, which is favorable to obtain high binding capacity and efficiency. Finally, SDS-HAc (2\%) was used to remove the embedded Lyz, leading to the preparation of recognition cavities complementary to template protein on the surface of MWCNTs-COOH@CS.

The morphology of Lyz-MIPs, NIPs and bare MWCNTs$\mathrm{COOH}$ were characterized using FETEM and FESEM. As shown in Fig. 2a and d, bare MWCNTs-COOH had very clean wall and central hollow tubular structure. The outer diameters of MWCNTs-COOH ranged from 20 to $30 \mathrm{~nm}$, and their lengths were tens of microns. After the coating process, Lyz-MIPs (Fig. 2b) exhibited clearly rough surface compared with bare MWCNTs-COOH, indicating the deposition of PDA thin film over the surface of the nanotube successfully. On the other hand, the FESEM of NIPs (Fig. 2c) had some slight adhesion among neighboring NIPs particles, implying that the absence of the template protein during synthesis process would affect the uniformity of PDA layer. From FETEM Fig. 2e and $\mathrm{f}$, it can be inferred that the diameter of Lyz-MIPs and NIPs became larger than that of the original MWCNTs-COOH (Fig. 2d), demonstrating the successful in situ spontaneous oxidative polymerization of dopamine on the surface of MWCNTs-COOH. The tubular layer of the highly uniformly coated PDA film was about $6 \mathrm{~nm}$, which can provide a high mass-transfer between the solution and the imprinted sites. From the FESEM and FETEM images, it could be inferred that the imprinted nano-film were successfully introduced on the surface of MWCNTs-COOH. Moreover, the BET specific surface area of Lyz-MIPs was 79.94 $\mathrm{m}^{2} \mathrm{~g}^{-1}$, while that of NIPs was only $68.80 \mathrm{~m}^{2} \mathrm{~g}^{-1}$. The pore volumes of Lyz-MIPs and NIPs were found to be 0.288 and 0.250 $\mathrm{cm}^{3} \mathrm{~g}^{-1}$, respectively. Compared Lyz-MIPs with NIPs, the parameters of MIPs were all higher than those of NIPs, demonstrating that Lyz-MIPs had more mesopores than NIPs. The differences in mesoporosity may be due to the variation of the polymerization process in the presence of template protein. 

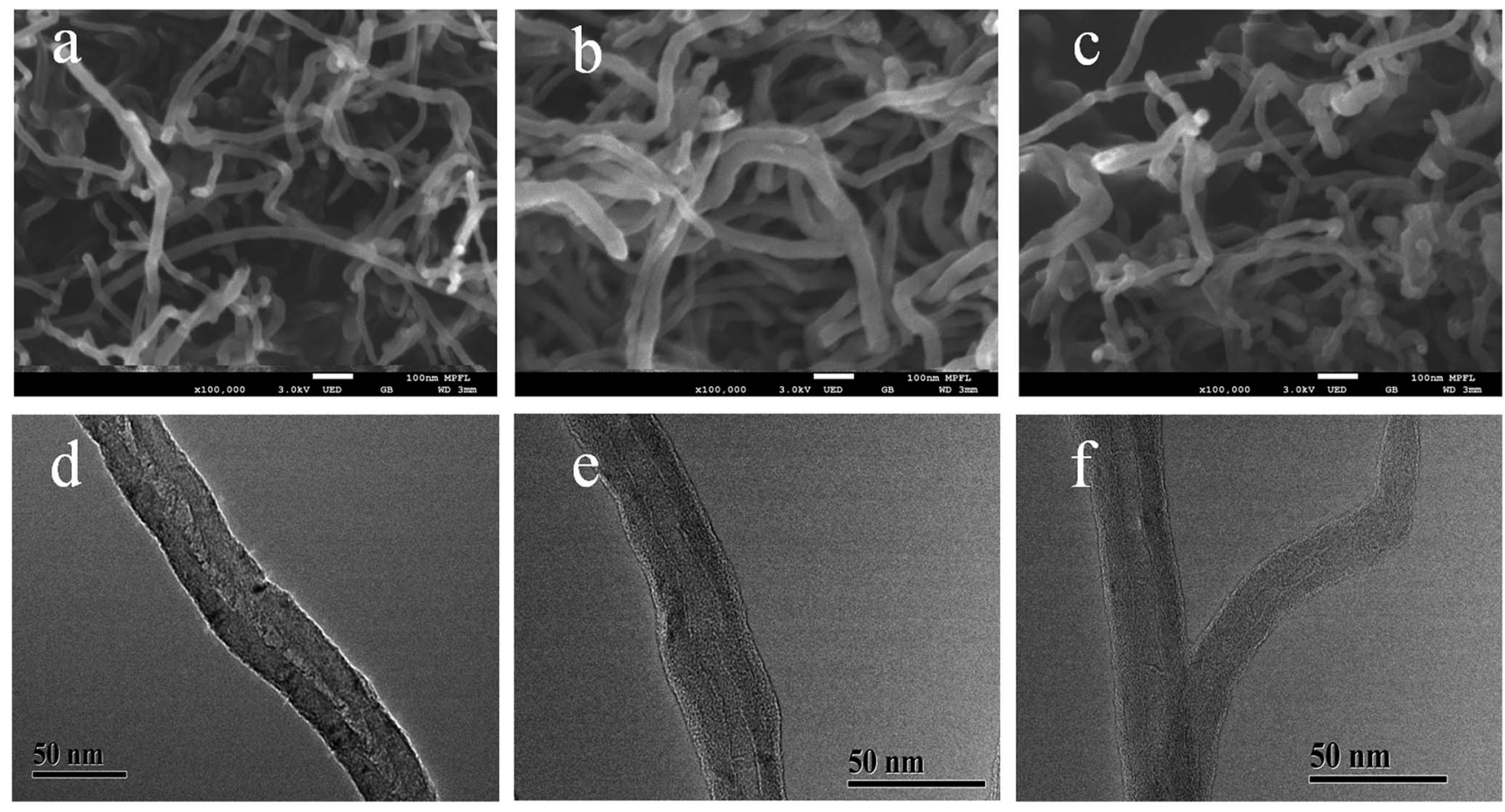

Fig. 2 FESEM and FETEM images of MWCNTs-COOH (a, d), Lyz-MIPs (b, e) and NIPs (c, f).

Thermogravimetric analysis (TGA) was performed to estimate the thermal stability of the prepared polymers. As indicated by the TGA results (Fig. S1†), when the temperature was below $200{ }^{\circ} \mathrm{C}$, a relatively low proportion of mass loss was observed for Lyz-MIPs, due to the evaporation of physically absorbed water. The result indicates the thermostability of LyzMIPs in normal use. To estimate the amount of PDA film attached on the surface of Lyz-MIPs, the TGA analysis of the naked MWCNTs-COOH and Lyz-MIPs were conducted. As shown in Fig. $\mathrm{S} 1, \dagger$ in the temperature range of $200-480{ }^{\circ} \mathrm{C}$, the mass loss ratio for MWCNTs-COOH was only about $4.83 \%$. While Lyz-MIPs and NIPs showed higher weight losses, which were about $24.69 \%$ and $16.50 \%$, respectively. From the stepwise increased weight loss results above, it can be deduced that the average mass content of PDA in imprinting polymers was about $17.82 \%$, demonstrating that the polymerization of dopamine was achieved.

Fourier transform infrared (FT-IR) spectra provided a direct proof for the deposition of PDA on the surface of MWCNTs$\mathrm{COOH}$ (Fig. S2 $\dagger$ ). The strong absorption peaks at approximately $3445 \mathrm{~cm}^{-1}(\mathrm{O}-\mathrm{H})$ and $1110 \mathrm{~cm}^{-1}(\mathrm{C}-\mathrm{O})$ were the characteristic peaks of the hydroxyl groups in the MWCNTs-COOH (Fig. S2a †). The peaks located at 1540 and $1398 \mathrm{~cm}^{-1}$ were corresponded to antisymmetrical vibration and symmetric vibration of $\mathrm{COO}^{-}$ respectively. The weak band appearing at $1750 \mathrm{~cm}^{-1}$ was attributed to stretching vibration of $\mathrm{C}=\mathrm{O}$, indicating that large amounts of carboxylate groups covered on the surface of MWCNTs-COOH. After coated by the polydopamine (Fig. S2b and $\mathrm{c} \dagger$ ), the bands at both 1506 and $1640 \mathrm{~cm}^{-1}$ were attributed to the superposition of phenylic $\mathrm{C}=\mathrm{C}$ stretching and $\mathrm{N}-\mathrm{H}$ bending, indicating the formation of polydopamine (PDA) layers.

\subsection{Optimization of preparation conditions}

The recognition capability of Lyz-MIPs is greatly dependent on polymerization process. In order to obtain the most favorable molecular polymers, different influencing factors, including the amount of chitosan mobilized on MWCNTs-COOH, the concentration of DA and polymerization time, were optimized respectively.

3.2.1 Effect of chitosan amount. In this work, different amounts of chitosan, ranging from 20 to $100 \mathrm{mg}$, were studied in the preparation process. Fig. 3a presents the results of the adsorption of Lyz-MIPs. The binding amount of Lyz-MIPs increased with the increasing amount of chitosan. We presumed that the modification could effectively improve the dispersion stability of MWCNTs-COOH in hydrophilic fluids. Moreover, the abundant amino and hydroxyl groups of chitosan increased the adsorption sites for target protein on the surface of MWCNTs-COOH. However, a continuous increase amount of chitosan will introduce too much amino groups, which obstructed the electrostatic interaction between the target protein and sorbents. At the chitosan amount of $40 \mathrm{mg}$, the best binding amount and imprinting factor for Lyz were obtained. In order to prove the functional modification effect of chitosan, examination of the adsorption capability between MWCNTsCOOH@MIPs (generated in the same way as Lyz-MIPs but without addition of CS) and Lyz-MIPs was carried out for comparison. The adsorption capacity of Lyz-MIPs (418 $\mathrm{mg} \mathrm{g}^{-1}$ ) was much higher than that of MWCNTs-COOH@MIPs (103 mg $\mathrm{g}^{-1}$ ), indicating that the chitosan plays an important role in the process of protein recognition. 

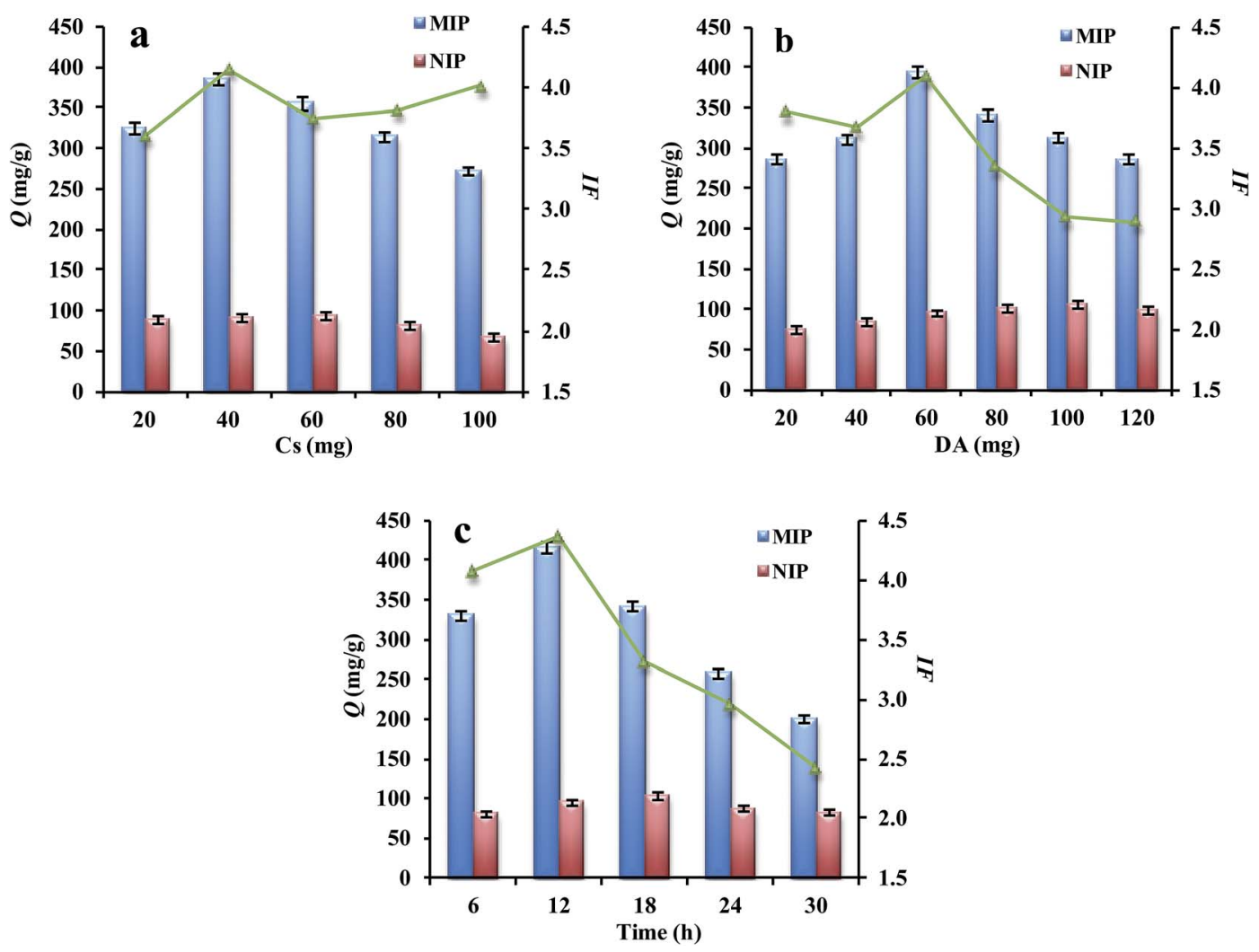

Fig. 3 Effect of the amount of CS (a), the amount of dopamine (b) and reaction time (c) on the adsorption performance of Lyz-MIPs and NIPs.

3.2.2 Effect of functional monomer (DA) amount. Similarly, the effect of DA concentration was also investigated in the range of $20-120 \mathrm{mg}$. As shown in Fig. 3b, when the mass of DA was increased from $20 \mathrm{mg}$ to $60 \mathrm{mg}$, both Lyz-MIPs and NIPs exhibited increased adsorption for Lyz, and the optimal mass was found to be $60 \mathrm{mg}$. The reason for this might be that higher amount of DA could increase the thickness of PDA layer and thus lead to the sufficient binding sites available for Lyz on the surface of MWCNTs-COOH. When the DA concentration was higher than $6 \mathrm{mg} \mathrm{mL}^{-1}$, the further increase of the thickness of PDA film caused the burying of recognition cavities and consequently leading to a high ratio of non-specific adsorption. The excessive functional monomer may also self-polymerize and block the recognition sites, resulting in low imprinting effect.

3.2.3 Effect of reaction time. In order to maximize the formation of imprinting cavities and minimize the generation of non-specific interactions, the effect of the polymerization time was finally investigated. As shown in Fig. 3c, with increasing the polymerization time to $12 \mathrm{~h}$, Lyz-MIPs exhibited the best binding amount and $I F$ among all the tested polymers, indicating that the optimal recognition sites and the best PDA layer thickness were formed at this condition. Further prolonging the self-polymerization time could lead to poor site accessibility for template protein, resulting in low imprinting factor. Therefore, the optimized preparation conditions were found to be $40 \mathrm{mg}$ of chitosan, $60 \mathrm{mg}$ of $\mathrm{DA}$, and $12 \mathrm{~h}$ of reaction time.

\subsection{Adsorption kinetics}

Fig. 4 shows adsorption kinetic curves for Lyz-MIPs and NIPs towards Lyz at $298 \mathrm{~K}$. In the initial stage, owing to the existence of large empty binding sites, the adsorption amount quickly increased. Then, with increasing the contact time, the effective binding sites were gradually captured and the adsorption equilibrium was slowly achieved after $40 \mathrm{~min}$. Moreover, LyzMIPs needed more time to reach the adsorption equilibrium,

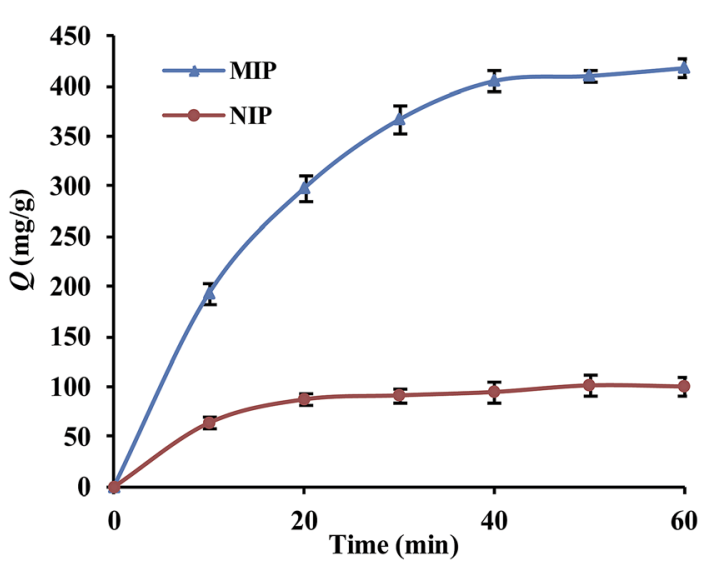

Fig. 4 The adsorption kinetics of Lyz on Lyz-MIPs and NIPs. Experimental conditions: $10 \mathrm{~mL}$ Lyz solution $\left(0.60 \mathrm{mg} \mathrm{mL}^{-1}, 50 \mathrm{mM}\right.$ Tris $-\mathrm{HCl}$ $\mathrm{pH}=8.5$ ) was incubated with $10 \mathrm{mg}$ polymers at $30{ }^{\circ} \mathrm{C}$. The points represent mean values of three measurements. 
which might be due to the abundant specific recognition process to Lyz through size and space effects.

In order to further validate the adsorption mechanism, the experiment data were tested using pseudo-first-order and pseudo-second-order kinetic equations, which were shown as follows, respectively:

$$
\begin{aligned}
& \ln \left(Q_{\mathrm{e}}-Q_{t}\right)=\ln Q_{\mathrm{e}}-k_{1} t \\
& \frac{t}{Q_{t}}=\frac{1}{k_{2} Q_{\mathrm{e}}{ }^{2}}+\frac{t}{Q_{\mathrm{e}}}=\frac{1}{v_{0}}+\frac{t}{Q_{\mathrm{e}}}
\end{aligned}
$$

where $Q_{\mathrm{e}}$ and $Q_{t}$ are the amount of adsorbate onto sorbent at the equilibrium and time $t(\mathrm{~min}) ; v_{0}\left(\mathrm{mg} \mathrm{g}^{-1} \mathrm{~min}^{-1}\right)$ is the initial adsorption rate; $k_{1}$ and $k_{2}$ are the equilibrium rate constants of pseudo-first-order and pseudo-second-order equation, respectively.

The related parameters were calculated and listed in Table 1. The $R^{2}$ values of the pseudo-second-order kinetic equation were higher than that of the pseudo-first-order kinetic equation, indicating that chemical process was the rate-limiting step during the adsorption. The $V_{0}$ of Lyz-MIPs (33.22 $\mathrm{mg} \mathrm{g}^{-1} \mathrm{~min}^{-1}$ ) is much higher than that of NIPs $\left(16.00 \mathrm{mg} \mathrm{g}^{-1} \mathrm{~min}^{-1}\right)$, indicating the presence of specific recognition sites on the surface of Lyz-MIPs. All the results suggested that the adsorption process was better described by the pseudo-second-order kinetic model.

\subsection{Adsorption isotherms}

The binding capacities for the tested templates of initial concentrations in the range of $0.10-0.70 \mathrm{mg} \mathrm{mL}{ }^{-1}$ were investigated for both Lyz-MIPs and NIPs. As observed from the isothermal curves (Fig. 5), the adsorption amount increased with the increase of the initial concentration and gradually reached the adsorption equilibrium. The amount of Lyz bounded to Lyz-MIPs ( $418 \mathrm{mg} \mathrm{g}^{-1}$ ) was about 4 times higher than that of NIPs (103 $\left.\mathrm{mg} \mathrm{g}^{-1}\right)$, suggesting that the specific recognition sites complemented to the template on spatial structure, molecular size and chemical effect were successfully formed on Lyz-MIPs. Comparatively, the adsorption of NIPs was dominated by the non-specific effect. Langmuir and Freundlich isotherm models were used to analyze the equilibrium values, which can be expressed by the following equations, respectively:

$$
\begin{gathered}
\frac{C_{\mathrm{e}}}{Q_{\mathrm{e}}}=\frac{1}{Q_{\mathrm{m}}} C_{\mathrm{e}}+\frac{1}{Q_{\mathrm{m}} K_{\mathrm{L}}} \\
\lg Q_{\mathrm{e}}=\lg K_{\mathrm{F}}+\frac{1}{n} \lg C_{\mathrm{e}}
\end{gathered}
$$

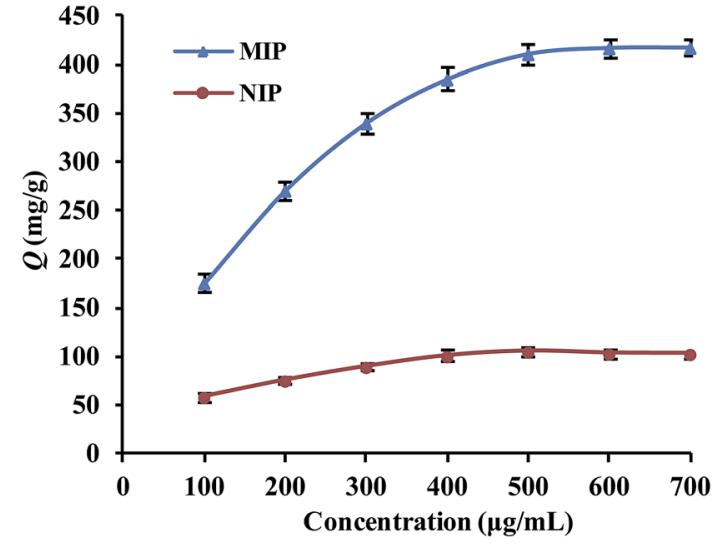

Fig. 5 The adsorption isotherms of Lyz on Lyz-MIPs and NIPs. Experimental conditions: $10 \mathrm{~mL}$ Lyz solution $\left(0.10-0.70 \mathrm{mg} \mathrm{mL}^{-1}\right.$, $50 \mathrm{mM}$ Tris $-\mathrm{HCl} \mathrm{pH}=8.5$ ) was incubated with $10 \mathrm{mg}$ polymers at $30{ }^{\circ} \mathrm{C}$. The points represent mean values of three measurements.

where $C_{\mathrm{e}}\left(\mathrm{mg} \mathrm{mL}^{-1}\right)$ is the equilibrium concentration of adsorbate; $Q_{\mathrm{e}}\left(\mathrm{mg} \mathrm{g}^{-1}\right)$ is the adsorption capacity at equilibrium; $Q_{\mathrm{m}}$ is the maximum adsorption capacity, theoretically; $K_{\mathrm{L}}$ $\left(\mathrm{mL} \mathrm{mg}^{-1}\right)$ and $K_{\mathrm{F}}\left(\mathrm{mg} \mathrm{g}^{-1}\right)$ are the Langmuir and Freundlich constant respectively; $1 / n$ is the Freundlich empirical constant that reflects the magnitude of the adsorption driving force.

The isotherm constants and liner correlation coefficients obtained from the experimental data are presented in Table 2. Through the comparison of liner correlation coefficients, the Langmuir isotherm model $(r>0.99)$ fitted the adsorption process of Lyz onto the polymers significantly better than the Freundlich isotherm model $(r<0.94)$. Therefore, the adsorption of Lyz onto Lyz-MIPs and NIPs may be a monolayer adsorption process.

Several imprinting methods have been reported in the literatures for specific binding of lysozyme. The imprinting factor and adsorption capacity $\left(Q_{\max }\right)$ of these literatures are summarized in Table 3. As can be seen, the Lyz-MIPs prepared in this work possessed remarkably higher $Q_{\max }$ and $I F$ than those of other literatures. ${ }^{35-45}$ Therefore, it is obvious that the method reported here has an enormous improvement.

\subsection{Adsorption specificity}

To evaluate the selectivity of Lyz-MIPs, besides the template lysozyme, four other proteins with a wide range of isoelectric

\begin{tabular}{|c|c|c|c|c|c|c|c|}
\hline Sorbents & \multicolumn{3}{|c|}{ Pseudo-first-order equation } & \multicolumn{4}{|c|}{ Pseudo-second-order equation } \\
\hline NIPs & 0.788 & 353.01 & 0.002 & 0.996 & 114.94 & $1.211 \times 10^{-3}$ & 16.00 \\
\hline
\end{tabular}
points (pI) and weight-average molecular weight $\left(M_{\mathrm{w}}\right)$ were chosen as control proteins to evaluate the selectivity of the

Table 1 Kinetic models and their statistical parameters

${ }^{a} Q_{\mathrm{e}, \mathrm{f}}\left(\mathrm{mg} \mathrm{g}^{-1}\right)$ is the calculated value of $Q_{\mathrm{e}}$ by Pseudo-first-order equation. ${ }^{b} Q_{\mathrm{e}, \mathrm{s}}\left(\mathrm{mg} \mathrm{g}^{-1}\right)$ is the caculated value of $Q_{\mathrm{e}}$ by Pseudo-second-order equation. 
Table 2 Parameters for Langmuir and Freundlich isotherm equations

\begin{tabular}{|c|c|c|c|c|c|c|c|}
\hline Sorbents & $Q_{\mathrm{e}, \mathrm{E}}^{a}\left(\mathrm{mg} \mathrm{g}^{-1}\right)$ & \multicolumn{3}{|c|}{ Langmuir isotherm equation } & \multicolumn{3}{|c|}{ Freundlich isotherm equation } \\
\hline NIPs & 103 & 0.994 & 119.05 & $11.85 \times 10^{-3}$ & 0.938 & 3.45 & 17.06 \\
\hline
\end{tabular}

${ }^{a} Q_{\mathrm{e}, \mathrm{E}}\left(\mathrm{mg} \mathrm{g}^{-1}\right)$ is the experimental value of $Q_{\mathrm{e}} \cdot{ }^{b} Q_{\mathrm{m}, \mathrm{L}}\left(\mathrm{mg} \mathrm{g}^{-1}\right)$ is the calculated value of $Q_{\mathrm{e}}$ by Langmuir isotherm equation.

prepared Lyz-MIPs. The pI and $M_{\mathrm{w}}$ of all the proteins studied are Lyz $\left(M_{\mathrm{w}} 14 \mathrm{kDa}, \mathrm{pI} 14.4\right)$, BSA $\left(M_{\mathrm{w}} 66.46 \mathrm{kDa}, \mathrm{pI} 4.7\right)$, pepsin $\left(M_{\mathrm{w}} 34.5 \mathrm{kDa}, \mathrm{pI} 1.0-2.5\right)$, $\mathrm{HSA}\left(M_{\mathrm{w}} 67 \mathrm{kDa}, \mathrm{pI} 4.64\right)$ and $\mathrm{BR}\left(M_{\mathrm{w}}\right.$ $33 \mathrm{kDa}$, pI 9.35), respectively. As shown in Fig. 6, it was obvious that Lyz-MIPs exhibited the highest adsorption capacity and imprinting factor to Lyz among these five proteins, where the selectivity coefficient of BSA, pepsin, HSA and BR were $4.30,7.12,3.06$ and 4.20 , respectively. The adsorption capacity of Lyz-MIPs and NIPs for BR was nearly $91 \mathrm{mg} \mathrm{g}^{-1}$, which was similar to $103 \mathrm{mg} \mathrm{g}^{-1}$ of NIPs toward Lyz and much higher than that for BSA and HSA proteins. This might be because the $M_{\mathrm{W}}$ of BSA and HSA are larger than that of BR, so these proteins were hard to enter into the recognition cavities on the surface of Lyz-MIPs. Moreover, according to the pI of Lyz and BR, they were positively charged in adsorption solution while DA was negatively charged during the adsorption process, while BSA and HSA were negatively charged. Thus, Lyz-MIPs could selectively adsorb more BR than BSA and HSA according to the theory of electrostatic interactions. As for pepsin, the pI is so low that will lead to a remarkable decline in electrostatic interaction between the protein molecules and the formed Lyz-MIPs. Therefore, the adsorption capacity of pepsin was the lowest among five proteins, although it has similar molecular size to Lyz. The above results indicate that the imprinted cavities with a combination of complementarities in molecular size, shape and multiple functional groups play an essential role in the specific recognition of template protein.

Table 3 Comparisonn of the adsorption capacity $\left(Q_{\max }\right)$ and imprinting factor (IF) with other published imprinted polymers for Lyz

\begin{tabular}{|c|c|c|c|}
\hline Polymers & $I F$ & $\begin{array}{l}Q_{\max } \\
\left(\mathrm{mg} \mathrm{g}^{-1}\right)\end{array}$ & Reference \\
\hline Lyz-imprinted cryogels & 3.9 & 10.3 & 35 \\
\hline Lyz-imprinted $\mathrm{Fe}_{3} \mathrm{O}_{4}$ nanoparticles & 1.5 & 67.6 & 36 \\
\hline $\begin{array}{l}\text { Lyz-imprinted magnetic } \\
\text { nanocomposites }\end{array}$ & 29.0 & 125.0 & 37 \\
\hline Lyz-imprinted silica nanoparticles & 4.0 & 53.3 & 38 \\
\hline Lyz-imprinted $\mathrm{Fe}_{3} \mathrm{O}_{4}$ nanoparticles & 2.0 & 8.3 & 39 \\
\hline Lyz-imprinted silica nanoparticles & 2.9 & 35.2 & 40 \\
\hline Lyz-imprinted magnetic nanoparticles & 3.7 & 250.0 & 41 \\
\hline Lyz-imprinted MCP beads & 2.5 & 31.7 & 42 \\
\hline Lyz-imprinted magnetic nanoparticles & 2.0 & 23.3 & 43 \\
\hline Lyz-imprinted $\mathrm{Fe}_{3} \mathrm{O}_{4}$ nanoparticles & 1.8 & 204.1 & 44 \\
\hline Lyz-imprinted silica nanoparticles & 3.1 & 49.6 & 45 \\
\hline Lyz-MIPs & 4.1 & 418.3 & This work \\
\hline
\end{tabular}

\subsection{Regeneration and reusability}

The regeneration ability is one of the key factors for the application performance of adsorbents. A batch adsorption experiment for Lyz-MIPs was firstly conducted to reach saturation adsorption. The Lyz-MIPs could be regenerated after washing with SDS-HAc (2\%). The experimental results are shown in Fig. 7. As can be seen, after six adsorption-desorption cycles, the adsorption capacity of the Lyz-MIPs for template protein was only reduced by $7.4 \%$. The possible reason is that some recognition cavities in the DA layer of Lyz-MIPs particles might be blocked after regeneration or be destroyed after rewashing/ centrifugation process, and thus they did not match with the template protein any more. Despite a small decreased, the

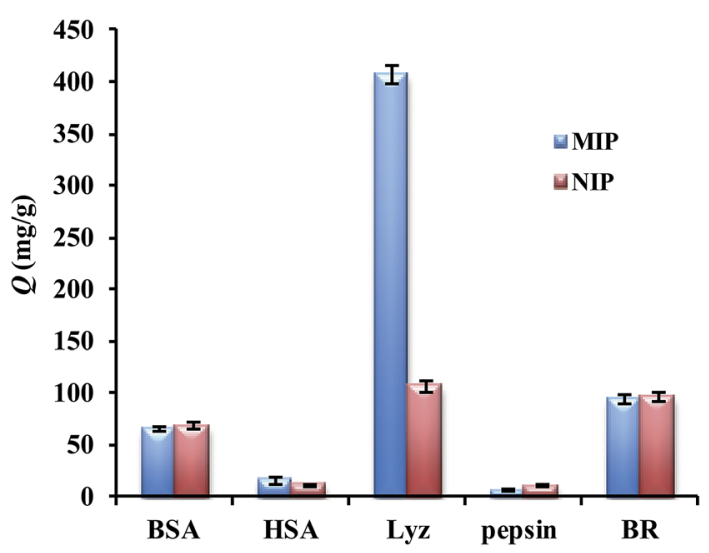

Fig. 6 The selective adsorption capacity of Lyz-MIPs and NIPS towards BSA, HSA, pepsin and BR.

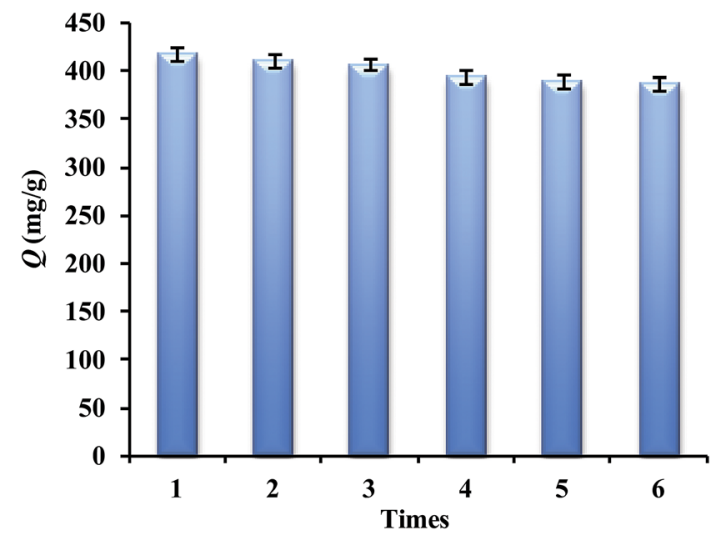

Fig. 7 Reusability of the Lyz-MIPs for six cycles. 


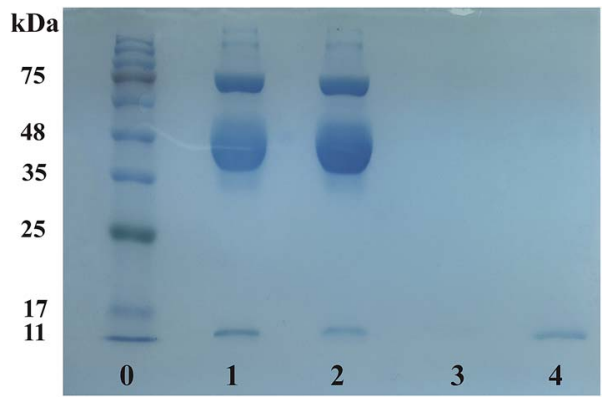

Fig. 8 SDS-PAGE analysis of chicken egg white before and after treatment with Lyz-MIPs and NIPs. Lane 0, marker; lane 1, fresh egg white diluted 10 -fold; lane 2, remaining egg white after adsorption by Lyz-MIPs; lane 3, the absorbed egg white by NIPs eluted with SDS-HAC (2\%); lane 4 , the absorbed egg white by Lyz-MIPs eluted with SDS-HAc (2\%).

absorption capacity of Lyz-MIPs was maintained 92.5\% after six cycles, exhibiting a good stability and regeneration performance. All these results confirm that the Lyz-MIPs prepared by this method is suitable for practical application.

\subsection{Real sample ananlysis}

The practical applicability of Lyz-MIPs was evaluated by direct purification of Lyz from the diluted egg white, and the results of SDS-PAGE are shown in Fig. 8. Compared with the 10-fold diluted egg white samples (lane 1), the intensity of Lyz band faded noticeably after being treated with Lyz-MIPs (lane 2). After elution with $0.1 \mathrm{M} \mathrm{NaCl}$, there was only one band reappeared clearly on $14.4 \mathrm{kDa}$ (lane 4), suggesting that Lyz was well isolated by prepared Lyz-MIPs and other proteins in the diluted egg white sample displayed no co-adsorption. In addition, the lysozyme molecules covered the same distance after the adsorption-elution process (lane 2 and 4), indicating that the efficient process did not denature the natural form of lysozyme structure. These results further confirm that the developed LyzMIPs with excellent imprinting effect and selectivity have obvious advantages for practical applications.

\section{Conclusions}

In this work, a facile and effective surface protein imprinting method through self-polymerization and nano-sized matrix for selective separation of proteins has been developed successfully. Comparing with previous works using Lyz as the template, our imprinted nanomaterials exhibit the biggest binding capacity, significant selectivity, and excellent reproducibility among these articles. Furthermore, the synthesis process is much simpler and is more suitable with the concept of "Green Chemistry", since we adopted MWCNTs-COOH as supports and DA as functional monomers. The practicability for biological application of as-prepared Lyz-MIPs was also evaluated by the successful usage in separating lysozyme from chicken egg white, showing potential values in industrial protein purification, basic biomedical research and clinical diagnostics. All these results indicated that the advantageous properties of this green method could significantly enhance the specific recognition ability of Lyz-MIPs and low the cost, thus this method would hold the potential to promote the development of molecular imprinting technique for protein.

\section{Acknowledgements}

This work was financially supported by National Natural Science Foundations of China (Grant No. 81573391 and 81173024), the National Key Projects of China (No. 81227802), China Postdoctoral Science Foundation (No. 2014M562428) and Shannxi Province Health Department Scientific Foundation (No. 2014D73). We are grateful to Dr Min Zhang and Prof. Ge Meng for revising the paper.

\section{References}

1 L. Sheng, J. Wang, M. J. Huang, Q. Xu and M. H. Ma, Int. J. Biol. Macromol., 2016, 92, 600-606.

2 T. T. Wu, C. H. Wu, S. L. Fu, L. P. Wang, C. H. Yuan, S. G. Chen and Y. Q. Hu, Carbohydr. Polym., 2017, 155, 192-200.

3 G. Bayramoglu, T. Tekinay, V. C. Ozalp and M. Y. Arica, J. Chromatogr. B: Anal. Technol. Biomed. Life Sci., 2015, 990, 84-95.

4 M. Mayani, C. D. M. Filipe and R. Ghosh, J. Membr. Sci., 2010, 347, 150-158.

5 B. Bobály, E. Tóth, L. Drahos, F. Zsila, J. Visy, J. Fekete and K. Vékey, J. Chromatogr. A, 2014, 1325, 155-162.

6 P. Q. Guo, Z. M. Luo, X. Y. Xu, Y. L. Zhou, B. L. Zhang, R. M. Chang, W. Du, C. Chang and Q. Fu, Food Chem., 2017, 217, 628-636.

7 P. Ma, Z. P. Zhou, J. D. Dai, L. Qin, X. B. Ye, X. Chen, J. S. He, A. Xie, Y. S. Yan and C. X. Li, RSC Adv., 2016, 6, 9619-9630.

8 C. Wang, Z. H. Guo, L. Zhang, N. Zhang, K. Y. Zhang, J. G. Xu, H. Y. Wang, H. W. Shi, M. Qin and L. Ren, RSC Adv., 2016, 6, 49597-49603.

9 P. Q. Guo, X. Y. Xu, L. Xian, Y. H. Ge, Z. M. Luo, W. Du, W. H. Jing, A. G. Zeng, C. Chang and Q. Fu, Talanta, 2016, 161, 830-837.

10 K. Hemmati, R. Sahraei and M. Ghaemy, Polymer, 2016, 101, 257-268.

11 W. Du, C. M. Lei, S. R. Zhang, G. Bai, H. Y. Zhou, M. Sun, Q. Fu and C. Chang, J. Pharm. Biomed. Anal., 2014, 91, 160168.

12 Z. Y. Yang, Q. Z. Cai, N. Chen, X. M. Zhou and J. L. Hong, RSC Adv., 2016, 6, 12193-12204.

13 Z. M. Luo, W. Du, P. Q. Guo, P. L. Zheng, R. M. Chang, J. Wang, A. G. Zeng, C. Chang and Q. Fu, RSC Adv., 2015, 5, 72610-72620.

14 R. Liu, M. Sha, S. S. Jiang, J. Luo and X. Y. Liu, Talanta, 2014, 120, 76-83.

15 J. Erdoessy, V. Horváth, A. Yarman, F. W. Scheller and R. E. Gyurcsányi, Trends Anal. Chem., 2016, 79, 179-190.

16 K. G. Yang, L. H. Zhang, Z. Liang and Y. K. Zhang, Anal. Bioanal. Chem., 2012, 403, 2173-2183. 
17 L. Qin, X. W. He, W. Zhang, W. Y. Li and Y. K. Zhang, Anal. Chem., 2009, 81, 7206-7216.

18 H. Y. Lin, C. Y. Hsu, J. L. Thomas, S. E. Wang, H. C. Chen and T. C. Chou, Biosens. Bioelectron., 2006, 22, 534-543.

19 G. Ertürk and B. Mattiasson, J. Chromatogr. B: Anal. Technol. Biomed. Life Sci., 2016, 1021, 30-44.

20 H. C. Chen, J. Kong, D. Y. Yuan and G. Q. Fu, Biosens. Bioelectron., 2014, 53, 5-11.

21 K. Y. Zhao, B. B. Lin, W. K. Cui, L. Z. Feng, T. Chen and J. F. Wei, Talanta, 2014, 121, 256-262.

22 Z. M. Luo, H. Shu, P. Q. Guo, P. L. Zheng, X. Y. Pan, W. Du, R. L. Liu, A. G. Zeng, C. Chun and Q. Fu, RSC Adv., 2016, 6, 15549-15557.

23 H. Nishino, C. S. Huang and K. J. Shea, Angew. Chem., Int. Ed., 2006, 45, 2392-2396.

24 Y. L. Wu, J. Zhao, C. Wang, J. Lu, M. J. Meng, X. H. Dai, Y. S. Yan and C. X. Li, Chem. Eng. J., 2016, 306, 492-503.

25 A. V. Herrera-Herrera, M. A. Gonzalez-Curbelo, J. HernandezBorges and M. A. Rodriguez-Delgado, Anal. Chim. Acta, 2012, 734, 1-30.

26 L. T. Yang, J. Yang, B. J. Xu, F. Q. Zhao and B. Z. Zeng, Talanta, 2016, 161, 413-418.

27 W. H. Chi, H. G. Shi, W. Shi, Y. Guo and T. Y. Guo, J. Hazard. Mater., 2012, 227-228, 243-249.

28 H. W. Wang, S. Yao, Y. Q. Liu, S. L. Wei, J. W. Su and G. X. Hu, Biosens. Bioelectron., 2017, 87, 417-421.

29 W. H. Wu, L. T. Yang, F. Q. Zhao and B. Z. Zeng, Sens. Actuators, B, 2017, 239, 481-487.

30 X. Yang, Z. H. Zhang, J. X. Li, X. Chen, M. L. Zhang, L. J. Luo and S. Z. Yao, Food Chem., 2014, 145, 687-693.
31 D. Zhang, D. J. Yu, W. J. Zhao, Q. Y. Yang, H. Kajiura, Y. M. Li, T. S. Zhou and G. Y. Shi, Analyst, 2012, 137, 26292636.

32 L. M. Wu, F. Liu, G. L. Wang, Z. A. Guo and J. C. Zhao, $R S C$ $A d v .$, 2016, 6, 464-471.

33 J. H. Kim, M. Lee and C. B. Park, Angew. Chem., Int. Ed., 2014, 53, 6364-6368.

34 Y. Q. Lv, T. W. Tan and F. Svec, Biotechnol. Adv., 2013, 31, 1172-1186.

35 K. Erol, K. KöSe, L. Uzun, R. Say and A. Denizli, Colloids Surf., $B, 2016,146,567-576$.

36 S. L. Ji, N. Li, Y. Shen, Q. Li, J. Qiao and Z. B. Li, Anal. Chim. Acta, 2016, 909, 60-66.

37 F. Lan, S. H. Ma, J. Ma, Q. Yang, Q. Y. Yi, Y. Wu and Z. W. Gu, Mater. Sci. Eng. C, 2017, 70, 1076-1080.

38 C. C. Yang, X. M. Yan, H. Guo and G. Q. Fu, Biosens. Bioelectron., 2016, 75, 129-135.

39 Q. Q. Gai, F. Qu, Z. J. Liu, R. J. Dai and Y. K. Zhang, J. Chromatogr. A, 2010, 1217, 5035-5042.

40 H. C. Chen, D. Y. Yuan, Y. Y. Li, M. J. Dong, Z. H. Chai, J. Kong and G. Q. Fu, Anal. Chim. Acta, 2013, 779, 82-89.

41 M. Zhang, X. H. Zhang, X. W. He, L. X. Chen and Y. K. Zhang, Nanoscale, 2012, 4, 3141-3147.

42 R. X. Gao, X. Kong, X. Wang, X. W. He, L. X. Chen and Y. K. Zhang, J. Mater. Chem., 2011, 21, 17863-17871.

43 N. Li, L. Qi, Y. Shen, J. Qiao and Y. Chen, ACS Appl. Mater. Interfaces, 2014, 6, 17289-17295.

44 L. Qin, X. W. He, X. Yuan, W. Y. Li and Y. K. Zhang, Anal. Bioanal. Chem., 2011, 399, 3375-3385.

45 H. C. Chen, J. Kong, D. Y. Yuan and G. Q. Fu, Biosens. Bioelectron., 2014, 53, 5-11. 